\title{
HYDRAULIQUE
}

\section{La Solution générale du Problème de la Détermination des Dimensions Economiques Maximum d'une Conduite forcée en Métal et son Application aux Calculs pratiques.}

Par PAur. P.-SANTO RINI, Ingénieur E. P. Z., Athènes.

Il est bien connu des ingénieurs hydrauliciens que la détermination des dimensions à donner aux éléments d'une conduite forcée en mélal, problème fondamental de la technique des aménagements hydro-électriques, acquiert une importance capitale en raison de la dépense afférente aux conduites sous pression d'une haule chute : en effet, les cas ne sont pas rares où cette dépense se chiffre à une fraction importante du coût de l'aménagement complet.

Précisons, que par le mot éléments nous désignons les inconnues du problème constructif qui sont en l'occurence les diamètres $D$ et les longueurs $l$ des tronçons successifs d'une conduite forcée de longueur totale connue $\mathrm{L}$.

La détermination des $(2 n-1)$ inconnues

$$
\left\{\begin{array}{lll}
\mathrm{D}_{1}, \mathrm{D}_{2}, & \ldots & \mathrm{D}_{\mathrm{n}} \\
l_{1}, l_{2}, & \ldots & l_{\mathrm{n}-1}\left({ }^{1}\right),
\end{array}\right.
$$

une fois le nombre $n$ de tronçons fixé ou déterminé a été souvent tentée mais jamais réalisée : comme conséquence, le problème de la détermination des dimensions économiques maximum d'une conduite fut considéré comme mathématiquement insoluble.

Afin d'obvier aux graves difticultés du problème mathématique, les divers auteurs ayant traité cette importante question ont toujours fait des hypothèses plus ou moins arbitraires.

Nous aussi, dans une étude parue en $1921\left({ }^{2}\right)$, nous avons adopté une condition pratique : celle d'imposer à priori des diamètres fixes correspondant à des tuyaux de fabrication courante.

Nous avons ainsi abouti à un nouveau procédé pour la détermination des points de séparation des tronçons successifs, l'emploi de nos tables $\left({ }^{3}\right)$ rendant, d'autre part, ces calculs simples et immédiats, facteurs justifiant la large adoption de notre méthode pour les applications pratiques $\left(^{4}\right)$.

(1) $l_{\mathrm{n}}$ n'est pas une inconnue, pouvant s'exprimer par

$$
l_{\mathrm{n}}=\mathrm{L}-\sum_{\mathrm{r}=1}^{\mathrm{r}=\mathrm{n}-1} l_{\mathrm{r}}
$$

(2) Papadopoulo-Santo Rini, ingénieur d'Etat à la Direction des Forces Hydrauliques, Athènes : "La Théorie du Rendement Economique Maximum d'une Conduite forcée en métal et le Calcul rationnel de ses Eléments ». ( La Houille Blanche ", juilletaoût 1921).

( ${ }^{3}$ P.-SAnto Rini : "I I Calcul rationnel des Eléments d'une Conduite forcée en mélal sur la base de son Rendement Economique Maximum ». (J. Rey, éditcur, Grenoble, 1921, p. 36 à 39).

( ${ }^{4}$ E. PAcoret : "Les Forces hydrauliques et les Usines hydroćlectriques" (Paris, 1923), pages 70 à 76 et 86 ;

"Génie Civil " (Paris, 1921), p. 298;

"La Houille Blanche" (Lyon, 1922), p. 227;

"Bulletin technique de la Suisse Romande" (Lausamne, 1923), p. 239 ;

"Bulletin d' Economie Hydrotechnique "(Prague, 1924), p. 13;

"Annali dei Lavori Pubblici" (Rome, 1924), p. 394; etc., etc.
Mais nous avons pensé que, du fait même de l'adoption des diamètres courants, la solution proposée ne peut être mathématiquement interprétée autrement que comme solution particulière du problème, la solution générale pouvant, d'autre part, fort bien conduire à une détermination encore plus rationnelle des éléments de la conduite.

C'est pourquoi, abandonnant dorénavant toute hypothèse arbitraire, nous exposerons dans ce mémoire aussi bien la solution générale du problème mathématique que son utilisation immédiate aux applications pratiques à l'aide d'un seul abaque graphique.

\section{I. - CONDUITE CURVILIGNE}

$\S$ I. - Exposé de la fonction minimum.

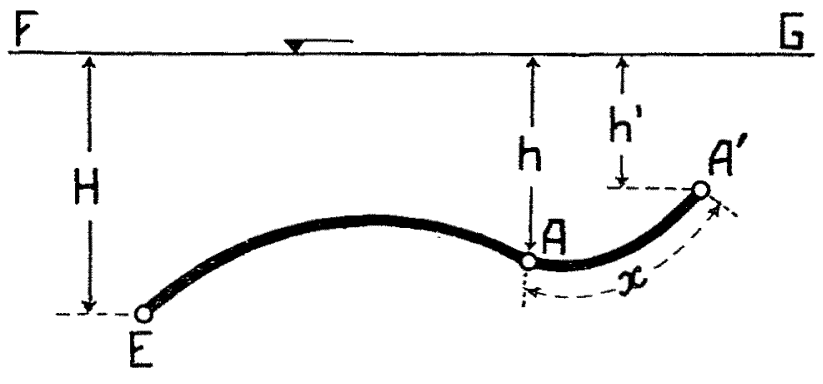

Fig. 1.

Soit A'E (Fig. 1) l'axe d'une conduite supposé curviligne dans son cas le plus général. Soient FG le niveau hydrostatique et $h$ ' et $\mathrm{H}$ les charges statiques à l'origine $\mathrm{A}$ ' et à l'extrémité $\mathrm{E}$ de la conduite de longueur $L\left({ }^{1}\right)$.

Supposons la courbe A'E définie par une relation entre la longueur $x$ de l'arc A'A et la charge hydrostatique $h$ correspondant à un point quelconque $\mathrm{A}$.

En résolvant cette relation par rapport à $h$,

$$
h=h(x)
$$

sera l'équation de la courbe A'E.

Nous avons déjà exposé dans notre monographie citée que la condition à satisfaire était celle de rendre maximum les bénéfices annuels $B$.

(1) $h^{\prime}=$ O O si A' lombe' sur le niveau hydrostatique F G. 
Pour exprimer ces bénéfices nous représenterons par :

E l'énergie électrique annuelle en $k W h$,

A le coût du $k W h$,

$P$ le poids de la conduite en tonnes,

$f$ le prix d'une tonne de conduite toute poséc,

$C$ les frais annuels d'exploitation el d'entretien,

$i$ le taux d'intérèt et d'amortissement, et

${ }^{v}$ le prix de vente du $k W h$.

Les frais anmuels seront alors représentés par

$$
\mathrm{V}=\mathrm{C}+(\mathrm{AE}+f \mathrm{P}) i
$$

alors que les revenus annuels s'exprimeront par

$$
\mathrm{R}=\nu \mathrm{E} \text {. }
$$

Les bénéfices anmuels $B$ seront done:

$$
\mathrm{B}=\mathrm{R}-\mathrm{V}=\mathrm{E}(v-\mathrm{A} i)-f \mathrm{P} i-\mathrm{C}
$$

En désignant encore par $q, w, s$ et $t$ des constantes connues ( ${ }^{1}$, ainsi que par :

$\mathrm{Q}$ le débit moyen annuel en $\mathrm{m}^{3} / \mathrm{sec}$,

H la charge statique à l'extrémité aval de la conduite en mètres, $d x$ l'élément infinitésimal de l'axe de la conduite $L$,

$\mathrm{Y}=q \mathrm{Q}^{2} \int_{0}^{L} \frac{d x}{D^{5}}$ la perte de charge lolale à l'exhémité de la conduite,

$e=w$ Dh l'épaisseur de paroi du luyau de diamètre D,

il résulte :

$$
\begin{aligned}
& \mathrm{E}=s \mathrm{Q}(\mathrm{II}-\mathrm{Y})=s \mathrm{Q} I \mathrm{I}-s q \mathrm{Q}^{3} \int_{0}^{\mathrm{L}} \frac{d x}{\mathrm{D}^{5}} \\
& \mathrm{P}=t \int_{0}^{\mathrm{I}} e \mathrm{D} d x=w t \int_{0}^{\mathrm{L}} \mathrm{D}^{2} h d x
\end{aligned}
$$

En substituant ces valeurs dans (1), on aura, enfin :

$$
\mathrm{B}=\text { constante }-\sum^{\prime} s q \mathrm{Q}^{3}(v-\mathrm{A} i) \int_{0}^{1} \frac{d x}{\mathrm{D}^{5}}+w t f i \int_{0}^{1} \mathrm{D}^{2} h d x
$$

Pour que B soit maximum, il suffit de rendre minimum l'expression entre parenthèses, ou ce qui revienl au même :

$$
\mathrm{K}_{\mathrm{u}}=\frac{2 \mathrm{~T}}{5} \int_{0}^{\mathrm{L}} \frac{d x}{\mathrm{D}^{5}}+\int_{0}^{\mathrm{L}} \mathrm{D}^{2} h d x
$$

(1)

$$
\begin{aligned}
q & =\frac{64 \vdots}{\pi^{2} c^{2}} \\
w & =\frac{i}{2 k} \\
s & =\frac{0,736 \cdot 1000}{75} a b \\
t & =\pi z p,
\end{aligned}
$$

les notalions étant les mêmes que dans notre mémoire cité, soient:

$\mu=$ coefficient exprimant le rapport de la perte de chargo totale de la conduite supposée mise en service, à la perte cle charge calculée simplement pour un tuyau d'axe rectiligne (p. ex. $3=1,05$ ),

$c=$ le coefficient de la formule de (hézy (p. ex. $c \quad . .60$ ),

$i=$ coefficient permettant de lenir compte de la surcharge slatique due au coup de bélier (p. ex. $;, \cdots 1,15$ ),

$\mathrm{k}=$ travail admis du métal à la traction en $t / \mathrm{m}^{2}$,

$=$ rendement de la soudure ou rivure $(p$. ex. $\because=0,90)$,

$a=$ le nombre d'heures de fonctionnement par année,

$b=$ rendement mécanique de l'installation (p. ex. $b=0,80$ ),

$z=$ coefficient exprimant le rapport du poids réel de la conduite toute posée, y compris les jonctions des tuyaux au poids calculé simplement d'après la formule approximative $e=w$ Dh (p. ex. $z=1,20$ ). en posant :

$$
\mathrm{T}=\frac{\left.5 s q \mathrm{Q}^{3}(v-\cdots) \Lambda i\right)}{2 v t f i}
$$

valeur qui peut ètre considérée comme une constante de l'améllagentent el jdentique à celle indiquée dans notre première étude.

\section{\$2. - Solution générale pour le cas d'une conduite à axe curviligne établie entièrement suivant la loi de la section aval.}

S'il eut été possible de construire une conduile à diamètres variables arec continuité de point en point, la loi de la variation de $\mathrm{D}$ s'obtiendrait immédiatement par la condition

fournissant l'expression

$$
\frac{a K_{u}}{a D}=0
$$

$$
\mathrm{D}=\sqrt[i]{\frac{\mathrm{T}}{h}}
$$

où $\mathrm{D}$ apparaît uniquement comme fonction de la charge statique $h$.

Dans l'impossibilité où l'on se trouve de construire une pareille conduite affeclant la forme d'un entonnoir, il est nécessiare de la remplacer par une aulre conduile constitue par $n$ troncons de diamètres constants $\mathrm{I}$.

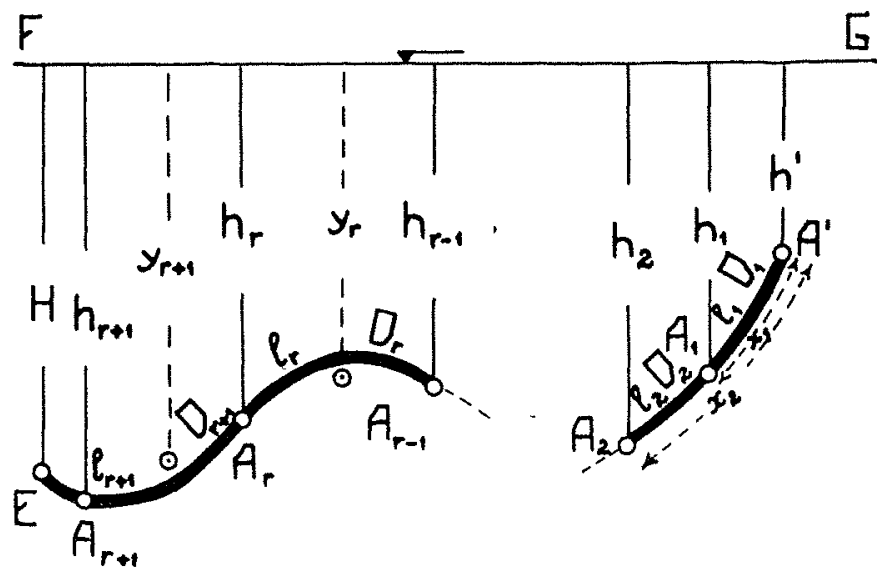

Fig. 2.

Ln désignant alors par $\Lambda_{r}$ le point de division entre les $r^{\text {me }}$ et $(r+1)^{\text {me }}$ tronçons, par $h_{\mathrm{r}}$ la charge slalique y relative, et par $l_{\mathrm{r}}$ et $\mathrm{D}_{\mathrm{r}}$ les longueur et diamètre du $r$ me troncon (Fig. 2), la fonclion $\mathrm{K}_{\mathrm{u}}$ tirée de (2) assume la forme :

$$
K_{u}=\frac{2 T}{5} \sum_{r=1}^{r=n} \frac{x_{r}-x_{r}-1}{D_{r}^{5}}+\sum_{r=1}^{r n} D_{i}^{n} \int_{x_{r-1}}^{x_{1}} h d x
$$

qui contient les $(2 n-1)$ inconnues

$$
\begin{aligned}
& \left.1 D_{1}, 1\right)_{2}, \ldots . D_{\mathbf{n}} \\
& i x_{1}, x_{2}, \ldots \ldots x_{n-1}
\end{aligned}
$$

Entre ces inconnues subsistent les $(2 n-1)$ relations qui s'obtiennent en annulant les dérivées partielles de $K$ par rapport aux D) et aux $x$.

En dérivant (4) par rapport à $D_{\mathrm{r}}$ el en amnulant celte dérivée, l'on obtient :

$$
-5 \cdot \frac{2}{5} \cdot T\left(x_{\mathrm{r}}-x_{\mathrm{r}-1}\right) \mathrm{I}_{1}^{-f i}+2 \mathrm{I}_{\mathrm{r}} \int_{x_{\mathrm{r}-1}}^{x_{\mathrm{r}}} h d x==0
$$


d'oùi :

$$
D_{1}=\sqrt{\int_{x_{\mathrm{r}-1}} h d x} \frac{\overline{\mathrm{T}\left(x_{\mathrm{r}}-x_{\mathrm{r}-1}\right)}}{x_{\mathrm{r}}}
$$

Si nous désiguons maintenant par $y_{\mathrm{r}}$ la charge slatique correspondant au centre de gravité du segment d'are $\left(x_{\mathrm{r}}-x_{\mathrm{r}-1}\right)$, il résulte :

$$
y_{\mathrm{r}}=\frac{\int_{x_{\mathrm{r}-1}}^{\cdot x_{\mathrm{r}}} h d x}{x_{\mathrm{r}}-x_{\mathrm{r}-1}},
$$

donc :

$$
D_{\mathrm{r}}=\sqrt[\pi]{\frac{\mathrm{T}}{y_{\mathrm{r}}}}
$$

L'expression (5) montre que les diamètres $D$ peuvent être déterminés lorsqu'on connaît la subdivision de la conduite en $n$ tronçons.

Pour établir cette subdivision et pour déterminer donc les (n-1) inconnues $x_{1}, x_{2}, \ldots x_{n-1}$, l'on pourrait annuler les dérivées partielles de (4) par rapport à ces inconnues et substituer ensuite aux D leurs valeurs fournies par (5). Pourtant, afin de simplifier les calculs, nous préférons substituer directement dans (4) les valeurs fournies par (5) et déterminer ensuite les valeurs de $x$ qui rendent minimum la nouvelle expression de $\mathrm{K}_{\mathrm{u}}$.

Cette substitution conduit à :

$$
\begin{aligned}
& \mathbf{K}_{\mathbf{u}}=\frac{2 \mathrm{~T}}{5} \sum_{\mathbf{r}=1}^{\mathbf{r}=\mathbf{n}}\left(x_{\mathrm{r}}-x_{\mathrm{r}-1}\right)\left(\frac{\mathrm{T}}{y_{\mathrm{r}}}\right)^{-\frac{5}{7}}+\sum_{\mathrm{r}=1}^{\mathrm{r}=\mathrm{n}}\left(\frac{\mathrm{T}}{y_{\mathrm{r}}}\right)^{\frac{2}{7}} \int_{x_{\mathrm{r}-1}}^{x_{\mathrm{r}}} h d x
\end{aligned}
$$

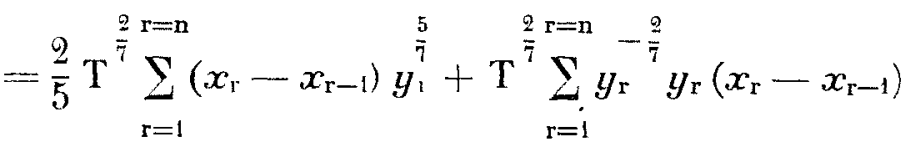

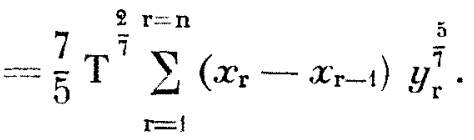$$
7 \mathrm{~T}^{\frac{9}{7}}
$$

$\frac{7}{5} \mathrm{~T}$ étant une constante, il s'ensuil que rendre minimim $\mathrm{K}_{\mathrm{u}}$ équivaut à rendre minimum la somme :

$$
\sum_{r=1}^{r=n}\left(x_{r}-x_{r-1}\right) y_{r}^{\frac{5}{7}}
$$

Nous pouvons donc énoncer le théorème suivant:

\section{THEOREME I}

l'our la subdivision là plus économique en n tronçons de n'importe quelle conduite forcée én métal d'épaisseur de paroi proportionnelle à la charge slatique el au diamètre en chaque point de la conduite, il est nécessaire et suffisant que la somme des moments du degré $\frac{5}{7}$ par rapport au niveau hydrostalique des longueurs des $n$ trongons supposées concentrées dans les centres de gravilé de ces tronçons, soit un minimum.
J'écuation simple :

$$
\sum_{r=1}^{r=n}\left(x_{r}-x_{r-1}\right) y_{r}^{\bar{i}}=\text { minimum }
$$

représente la condition essentielle du problème examiné.

Nous montrerons maintenant comment la relation (6) fournit les $(n-1)$ équations susceptibles de déterminer les $(n-1)$ inconnues $x_{1}, x_{2}, \ldots . x_{n-1}$.

A cet effet, et en annulant la dérivée partielle de (6) par rapport à $x$, on oblient:

$$
\begin{gathered}
\frac{5}{\overline{7}}\left(x_{\mathrm{r}+1}-x_{\mathrm{r}}\right) y_{\mathrm{r}-1}^{-\frac{2}{\overline{7}}} \frac{d y_{\mathrm{r}+1}}{d x_{\mathrm{r}}}-y_{\mathrm{r}+1}^{\frac{5}{\overline{1}}}+\frac{5}{\overline{7}}\left(x_{\mathrm{r}}-x_{\mathrm{r}-1}\right) y_{\mathrm{r}}^{-\frac{2}{4}} \frac{d y_{\mathrm{r}}}{d x_{\mathrm{r}}} \\
\ldots-y_{\mathrm{r}}^{\overline{\bar{T}}}=0 .
\end{gathered}
$$

Or :

$$
\begin{aligned}
& \quad \int_{d \frac{x_{\mathrm{r}}}{x_{\mathrm{r}+1}} h d x}^{x_{x_{\mathrm{r}}+1}}\left(x_{\mathrm{r}+1}-x_{\mathrm{r}}\right) \frac{d}{d x_{\mathrm{r}}} \int^{x_{\mathrm{r}+1} h d x+\int_{x_{\mathrm{r}}} h d x} x^{x_{\mathrm{r}+1}} \\
& \frac{d y_{\mathrm{r}+1}}{d x_{\mathrm{r}}}=\frac{x_{\mathrm{r}+1}-x_{\mathrm{r}}}{d x_{\mathrm{r}}}=\frac{x_{\mathrm{r}}}{\left(x_{\mathrm{r}+1}-x_{\mathrm{r}}\right)^{2}} \\
& =\frac{-\left(x_{\mathrm{r}+1}-x_{\mathrm{r}}\right) h_{\mathrm{r}}+\left(x_{\mathrm{r}-1}-x_{\mathrm{r}}\right) y_{\mathrm{r}+1}}{\left(x_{\mathrm{r}+1}-x_{\mathrm{r}}\right)^{2}}=\frac{y_{\mathrm{r}+1}-h_{\mathrm{r}}}{x_{\mathrm{r}+1}-x_{\mathrm{r}}}
\end{aligned}
$$

et, d'une manière analogue :

$$
\frac{d y_{\mathrm{r}}}{d x_{\mathrm{r}}}=\frac{h_{\mathrm{r}}-y_{\mathrm{r}}}{x_{\mathrm{r}}-x_{\mathrm{r}-1}}
$$

En substituant, l'on obtient enfin :

$$
-y_{\mathrm{r}+1}^{\frac{5}{7}}+\frac{5}{7} y_{\mathrm{r}+1}^{-\frac{2}{7}}\left(y_{\mathrm{r}+1}-h_{\mathrm{r}}\right)+\frac{-5}{7}^{-\frac{5}{7}}+\frac{5}{7} y_{\mathrm{r}}^{-\frac{2}{7}}\left(h_{\mathrm{r}}-y_{\mathrm{r}}\right)=0
$$

d'où :

$$
\frac{2}{5} y_{\mathrm{r}+1}^{\frac{5}{7}}+y_{\mathrm{r}+1}^{-\frac{9}{7}} h_{\mathrm{r}}=\frac{2}{5} y_{\mathrm{r}}^{\frac{5}{7}}+y_{\mathrm{r}}^{-\frac{2}{7}} h_{\mathrm{r}}
$$

qui est l'équation fondamentale à l'aide de laquelle se déterminent les longueurs des tronçons.

$y_{\mathrm{r}-1}$ étant fonction de $x_{\mathrm{r}+1}$ et $x_{\mathrm{r}}, y_{\mathrm{r}}$ fonction de $x_{\mathrm{r}}$ et $x_{\mathrm{r}-1}$, et $h_{\mathrm{r}}$ fonction de $x_{\mathrm{r}}$, l'équation (7) ne contient que les seules trois inconnues successives $x_{\mathrm{r}+1}, x_{\mathrm{r}}$ et $x_{\mathrm{r}-1} ; r$ pouvant assumer les valeurs de 1 à $(n-1)$, le nombre des équations (7) sera également $(n-1)$. La première de ces équations ne contiendra que les deux inconnues $x_{1}$ et $x_{2}$, et, d'une manière analogue, la dernière équation contiendra seulement les deux inconnues $x_{n-2}$ et $x_{n-1}$.

La résolution du système des $(n-1)$ équations $(7)$ entre les $(n-1)$ inconnues $x_{1}, x_{2}, \ldots . x_{n-1}$ devient donc évidente : la première équation fournira $x_{2}$ en fonction de $x_{1}$, la deuxième $x_{3}$ en fonction de $x_{2}$ et $x_{1}$, donc, implicitement, de $x_{1}$, et ainsi de suite jusqu'à l'avant-dernière équation qui fournira $x_{n-1}$ en fonction de $x_{1}$. En substituant les valeurs obtenues de $x_{n-1}$ et $x_{n-2}$ dans la dernière équation, celle-ci ne contiendra plus que la seule variable $x_{1}$ qui sera ainsi déterminée.

Les longueurs des tronçons de la conduite élant déterminées à l'aide des équations (7), les diamètres s'obtiennent immédiatement par les équations (5).

En ce qui concerne la valeur la plus rationnelle pour le nombre de tronçons $n$, l'on obtient facilement cetle détermination en faisant intervenir dans les calculs le coût d'un changement de diamètre. Un des paragraphes suirants sera consacré au calcul de $n$. 


\section{\$3. - Solution générale pour le cas d'une conduite à axo} curviligne composée d'une section aval et d'une section amont.

Dans la résolution du problème étudié au paragraphe précédent, nous avons admis que l'épaisseur de paroi varie suivant la loi

$$
e=w \mathrm{D} h
$$

où $w$ représente une constante.

Pratiquement, l'épaisseur de paroi ne peut descendre au-dessous d'une certaine valeur limite $e_{0}$ que nous avons définie, à la page 19 de notre monographie citée, par

$$
e_{\mathrm{o}}=\delta \mathrm{D}
$$

où ¿ représente une constante voisine de 0,005 .

En désignant par $h_{0}$ la charge statique correspondant au point $A_{0}$ de la conduite (Fig. 3 ) où l'épaisseur de paroi a la valeur limite $e_{\mathrm{o}}$, on a :

$w \mathrm{D} h=\vdots \mathrm{D}$

d'où :

$$
h_{\mathrm{o}}=\frac{\partial}{u}
$$

donc :

$$
h_{\mathrm{o}}=\text { constante constructive de la conduite. }
$$

Le point $A_{0}$ vient ainsi diviser la conduile en deux sections, celle aval $\mathrm{A}_{0} \mathrm{E}$ où l'épaisseur de paroi varie avec continuité suivant la loi $e=w \mathrm{D} h$ et celle amont $\mathrm{A}_{\mathrm{o}} \mathrm{A}^{\prime}$ où l'épaisseur de paroi est

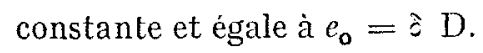

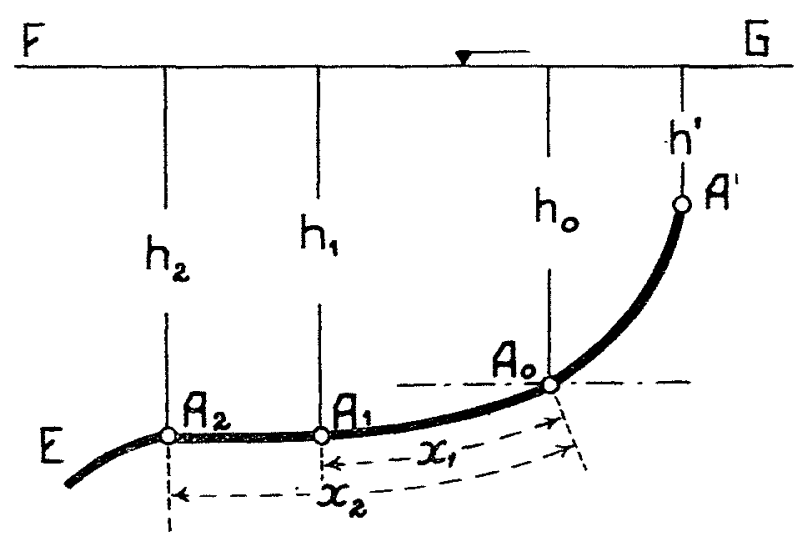

Fig. 3.

Ceci établi, passons à la résolulion malhémalique du problème relatif au calcul des éléments d'une conduite forcée ainsi définie, c'est-à-dire composée d'une section aval et d'une section amont.

Désignons à cet effet par $\mathrm{L}_{\mathfrak{u}}$ la longueur de la section aval, par $l_{0}$ celle de la section amont, par $D_{o}$ le diamètre en cette section, et choisissons le point $\mathrm{A}_{0}$ comme origine des coordonnées $x$.

En répétant le calcul des bénéfices annuels B, ceux-ci s'expriment ici par :

$$
\begin{gathered}
\mathrm{B}=\text { constante }-\left\{s q \mathrm{Q}^{3}(v-\lambda i)\left[\int_{0}^{\mathrm{L}_{\mathrm{u}}} \frac{d x}{\mathrm{D}^{5}}+\frac{l_{0}}{\mathrm{D}_{0}^{\pi}}\right]\right. \\
\ldots+w t f i \int_{0}^{\mathrm{L}_{u}} \mathrm{D}^{2} l l d x+k t f i \mathrm{D}_{0}^{2} l_{\mathrm{o}}
\end{gathered}
$$

Conme dans le cas du paragraphe 1, l'expression entre parenthèses doit être mininum afin que $\mathrm{B}$ devienne maximum, donc :

$$
\mathrm{K}=-\frac{2 \mathrm{~T}}{5} \int_{i}^{\mathrm{L}} \frac{d x}{\mathrm{D}^{5}}+\int_{i}^{\mathrm{I}} \mathrm{D}^{2} h d x+\frac{2 \mathrm{~T}}{5} \frac{l_{\mathrm{o}}}{\mathrm{D}_{\mathrm{o}}^{5}}+h_{\mathrm{o}} l_{\mathrm{o}} \mathrm{D}_{\mathrm{o}}^{2}
$$

où la constante $\mathrm{T}$ a toujours la mème valeur (3).

En confrontant l'expression (8) de K pour une conduite constituée par deux sections : amont et aval, avec l'expression (2) établie pour le cas d'une conduite dépourvue de seclion amont, l'on peut écrire :

$$
K=K_{u}+K_{m}
$$

où par $K_{\mathrm{m}}$ nous désignons la somme :

$$
K_{\mathrm{m}}=\frac{2 \mathrm{~T}}{5} \frac{l_{0}}{\mathrm{D}_{\mathrm{o}}^{5}}+h_{\mathrm{o}} l_{\mathrm{o}} \mathrm{D} \mathrm{o}_{\mathrm{o}}^{2}
$$

En derivant maintenant $K$ par rapport à $1 \omega_{0}$ et en annulant celte dérivée, l'on a :

$$
\frac{d \mathrm{~K}}{d \mathrm{D}_{\mathrm{o}}}=\frac{d \mathrm{~K}_{\mathrm{m}}}{d}=-5 \cdot \frac{2}{\overline{\mathrm{D}}} \cdot \mathrm{T} l_{\mathrm{o}} \mathrm{D}_{\mathrm{o}}^{-6}+2 h_{\mathrm{o}} l_{\mathrm{o}} \mathrm{D}_{\mathrm{o}}=0
$$
d'où :

$$
D_{11}=V^{7}: \frac{T}{h}
$$

qui fournit la valeur conslante du diamètre $\mathrm{D}_{\mathrm{o}}$ pour lu section amont.

Observant ensuite que $\mathrm{K}_{\mathrm{m}}$ est indépendant des variables $x$ el $\mathrm{D}$ qui n'apparaissent qu'en $K_{u}$, il résulle évidemment que :

$$
\begin{aligned}
& \frac{d \mathrm{~K}}{d \mathrm{D}}=\frac{d \mathrm{~K}_{\mathrm{u}}}{d \mathrm{D}} \\
& \frac{d \mathrm{~K}}{d x}=\frac{d \mathrm{~K}_{\mathrm{u}}}{d x}
\end{aligned}
$$

D'où la conclusion :

La solution indiquée au paragraphe 2 pour une conduite dépourvue de section amont est aussi valable pour la subdivision de la section aval d'une conduite pourvue de section amont.

En particulier, le théorème I énoncé au paraple 2 se modifie en :

\section{THEOREME II}

Le point de subdivision d'une conduite forcée en métal en une section amont et en une section aval est caractérisé par une chairge statique dont la valeur constante est indépendunte de l'aménagement en étude. La section amont se compose d'un scul tronçon. Pour la subdivision la plus économique en n tronçons de la section aval, il est nécessaire et suffisant que la somme des moments du degré tique des longlueurs des $\mathbf{n}$ tronçons supposées concentrées dans les centres de gravité de ces tronçons, soit un minimum.

Pour la section aval supposée divisée en $n$ tronçons, la condition

$$
\sum_{r=1}^{r=n}\left(x_{r}-x_{r-1}\right) y_{r}^{\frac{5}{7}}=\text { minimum }
$$

doit être vérifiée, condition qui fournit le système de relations dérivées, servant au calcul des longueurs des Ironçons: 
Pour la section aval, les diamètres se déterminent par l'expression :

(5) :

$$
D_{\mathrm{r}}=\sqrt[7]{\frac{\mathrm{T}}{y_{\mathrm{r}}}}
$$

Par contre, pour la section amont, celle-ci n'est conslituée que d'un seul tronçon donl le diamètre est fourni par:

(9) :

$$
D_{\mathrm{o}}=\sqrt[7]{\frac{\mathrm{T}}{h_{\mathrm{o}}}}
$$

où nous rappelons que $h_{0}$ est la charge statique du point de séparalion entre les sections aval et amont, valeur pratiquement voisine de 100 mètres.

Il est à observer que la longueur et la configuration de la section amont n'ont aucune influence sur les résultats que nous venons d'indiquer.

L'axe de la conduite étant connu, on commence par tracer la parallèle $\mathrm{A}_{\mathrm{o}} \mathrm{S}$ (Fig. 4) située à $h_{0}$ en dessous du niveau hydrostatique $\mathrm{FG}$.

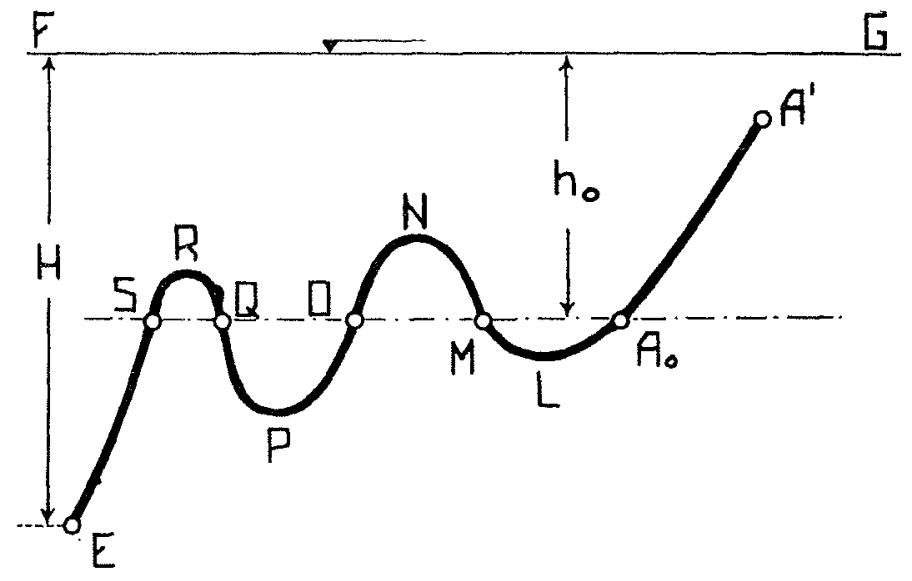

Fig. 4.

En ce qui concerne les segments de conduite aut-dessus de $A_{0} S$ Ia relation :

$$
o=\frac{d \mathrm{~K}}{d \mathrm{D}_{\mathrm{o}}^{\prime}}=\frac{d \mathrm{~K}_{\mathrm{m}}^{\prime}}{d \mathrm{D}_{\mathrm{o}}^{\prime}}=\frac{d}{d \mathrm{D}_{\mathrm{o}}^{\prime}}\left(\frac{2 \mathrm{~T}}{\hbar} \frac{l_{\mathrm{o}}}{\mathrm{I}_{\mathrm{o}}^{\prime}}+h_{\mathrm{o}} l_{\mathrm{o}} \dot{\mathrm{D}}_{\mathrm{o}}^{2}\right)
$$

fournit la valeur

$$
\mathrm{D}_{\mathrm{o}}^{\prime}=\downarrow^{i} \frac{\mathrm{T}}{h_{\mathrm{o}}}
$$

L'une maniere analogue:

fournil la valeur

$$
o=\frac{d \mathrm{~K}}{d \mathrm{D}_{\mathrm{o}}^{\prime \prime}}=\frac{d \mathrm{~K}_{\mathrm{m}}^{\prime \prime}}{d \mathrm{D} \mathrm{m}_{\mathrm{o}}^{\prime \prime}}
$$

et ainsi de suite.
Par conséquent :

Toutes les portions de conduite situées au-dessus de $\Lambda_{0} \mathrm{~S}$ ont toujours le même diamètre constant

$$
\mathrm{D}_{\mathrm{o}}=\sqrt[7]{\frac{\mathrm{T}}{h_{0}}}
$$

En ce qui est des segments de conduite situés au-dessous de $A_{o} S$, l'on peut remarquer que les termes $K_{u}$ sont indépendants entre eux; il résultera donc

$$
\mathrm{K}_{\mathrm{m}}=\text { minimum }
$$

si l'on aura simullanément:

$$
\begin{gathered}
\mathrm{K}_{\mathrm{u}}=\text { minimum } \\
\mathrm{K}_{\mathrm{u}}^{\prime \prime}=\operatorname{minimum} \\
\ldots \ldots \ldots \ldots \\
\text { elc. }
\end{gathered}
$$

Il s'ensuit que :

La subdivision des portions de conduäte situées au-dessous de $\mathrm{A}_{0} \mathrm{~S}$ devra s'effectuer l'une indépendamment de l'autre.

Pour chacune de ces portions subsiste la relation fondamentale:

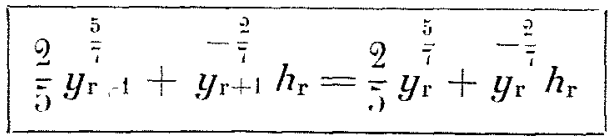

qui permet la subdivision de la conduite en un nombre $n$ de tronçons, la valeur de $n$ se déterminant en faisant intervenir la dépense afférente à un changement de diamètre.

Pour chacun de ces tronçons le diamètre se détérmine loujours par, la même formule

$$
\mathrm{D}_{\mathrm{r}}=\sqrt{\frac{\mathrm{T}}{y_{\mathrm{r}}}}
$$

Le problème mathématique de la détermination des éléments d'une conduite forcée curviligne se trouve donc complètement résolu.

En ce qui est des applications pratiques, la résolution mathématique des relations (7) présuppose évidemment la connaissance de l'équation algébrique de la courbe formée par l'axe de la conduite. Or, cet axe étant supposé suivre les sinuosités du terrain, l'équation qui en résulte peut affecter une forme des plus compliquées, rendant de ce fait mème la résolution des équalions (7) très laborieuse, sinon inpossible. Deux procédés peuvent donc être envisagés pour tourner celle difficulté :

10 En utilisant la propriété géométrique exposée dans le théorème II énoncé au paragraphe 3 , l'on peut parvenir après quelques essais à une bonne subdivision de la conduite,

$2^{\circ}$ En décomposant le tracé curviligne en un tracé polygonal dont nous montrerons la détermination automatique. Cette solution que nous recommandons pour les applications pratiques permet la résolution immédiate du problème. Il nous a été, en effet, possible d'étendre aux conduites polygonales un procédé graphique de calcul dérivant de l'application des formules de ce chapitre au cas des tronçous rectilignes compasant un tracé polygonal.

\section{(A suivre.)}




\title{
Sur une formule de perte de charge d'application universelle
}

\author{
Par M. A. Hancienser, Ingénielu de's Ponls el Chaussées.
}

La question suivante se pose sourent pour les ingénieurs hydrauliciens, qu'il s'agisse du calcul d'une conduite forcée, d'un canal d'amenée, ou encore d'un calcul d'évacuation des crues :

Quelle formule de perte de charge dois-je appliquer?

Nombreuses - et parfois àssez discordantes - sont, en effet, les formules indiquées par les expérimentateurs et les auteurs qui se sont spécialisés dans l'étude de l'hydraulique appliquée. On trouverait facilement, dans les traités d'hydraulique 80 formules, ou davantage, reposant sur un nombre plus ou moins grand d'expériences, entre lesquelles le choix est parfois malaisé, d'autant que la plupart des expériences exécutées l'ont été à l'échelle du laboratoire, et que force est souvent de se livrer à des extrapolations hasardeuses avant de les appliquer.

Aussi doit-on être reconnaissant à tous ceux qui essaient, en reprenant les matériaux déjà réunis, et au besoin en en apporlant de nouveaux, de présenter en quelque sorle la synthèse des résultats acquis, et de fournir des éléments d'information précis.

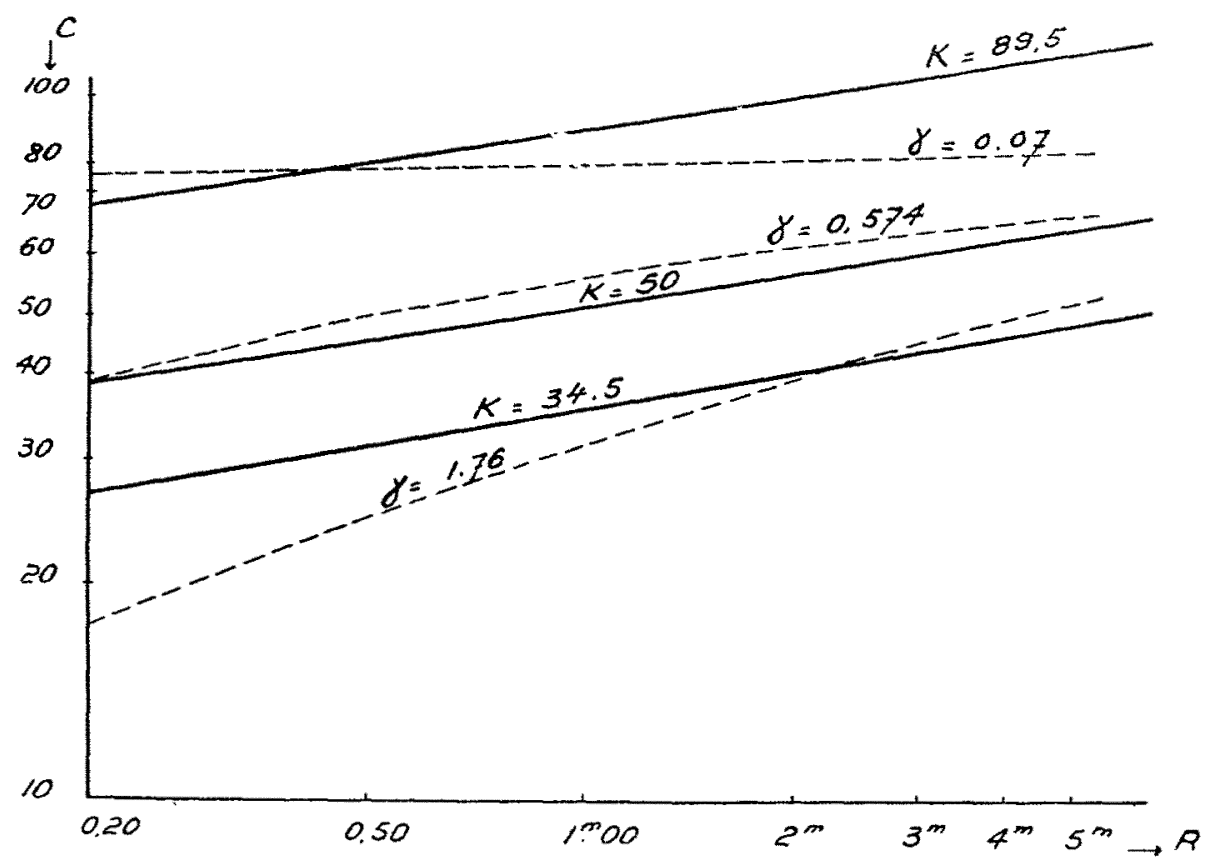

Fig. 1.- Variations du cocfficient $c$ da Chezy. Formule proposée : $c=-\mathrm{K} R \mathrm{R} / \mathrm{f}$. Formule de Bazin $c=\frac{8 i}{1-\frac{i}{1 / \bar{R}}}$ (Diagramme logarithmique. Ei abscisse : le rayon moyen; $(n$ orronnée : le coefficient $c$ ).

Nous nous proposons, dans cette courle note, de faire connaître à nos collègues français, ingénieurs et hydrauliciens, une récente et remarquable étude de cette nature, due à M. Strickler, ingénieur au Service des Eaux Suisse, et publiée par ce Service (1). Nous aurons en même temps l'occasion de prendre connaissance de quelques résultats intéressants signalés par cet auteur.

(1) Beitregge zur lirage der Geschwindigkeitsformeln und des Rauh!gkeitszahlen fur Ströme, Kanale und Geschlossene Leitungen. - Berne 1923.
Les tormules de perte de charge actuellement les plus usitées sont les suivantes, qui dócoulent lontes de la formule de Chézy :

$$
u=c l \cdot \overline{R j} \text {. }
$$

\section{A. Canuux découverts el rivières.}

Formule française de Bazin :

$$
u=\frac{87}{1+\frac{\gamma}{V \bar{R}}}, \overline{R j}
$$

Formule suisse de Canguillet et Kutter :

$$
u=\frac{03+\frac{1}{n}+\frac{0,00155}{j}}{1+\left(2 n+\frac{0,00155}{j}\right) \frac{n}{V^{\prime} \overline{\mathrm{K}}}} \times \downarrow \overline{\mathrm{R} j}
$$

Formule simplifiée de Kutter.

$$
u=\frac{100 V \overline{\mathrm{R}}}{m+V \overline{\mathrm{R}}} \times \overline{\mathrm{R} j}
$$

1)ans ces formules, où on prend comme unilés le mètre et la seconde, y désigne la perte de charge, u la vitesse movenne, Is le rayon hydraulique moyen; les coefficients $\%, n$, $m$ varienl avec $\mathrm{l}^{\text {a }}$ rugosité des parois moullées.

\section{B. Conduites forcées.}

Pour les luyaux métalliques et les conduites forcées, on ulilise habituellement des formules spéciales, dont la plus connue est celle de Maurice Lévy :

$$
u=\mathrm{A} V \overline{\frac{\mathrm{D}}{2} j\left(1+3 \sqrt{\left.\frac{\mathrm{D}}{2}\right)}\right.}
$$

dans laquelle 1) représente le diamètre, et le coefficient $A$ ayant pour valeur 36,4 s'il s'agit de tuyaux neufs, et 20,5 , s'il s'agit de tuyaux en fonte chargés de dépôts. Pour les conduites en bélon armé, on prend généralement $\Lambda$ égal à 25 ou à 28 .

M. l'Ingénieur Strickler propose de revenir à l'ancienne formule de Crauckler :

$$
u=k \mathrm{I}^{\stackrel{2}{3}} j^{2}
$$

le coefficient $k$ caractérisant la nature des parois (1).

(1) I.e coefficient $k$ aurait luimêne pour expression, d'après

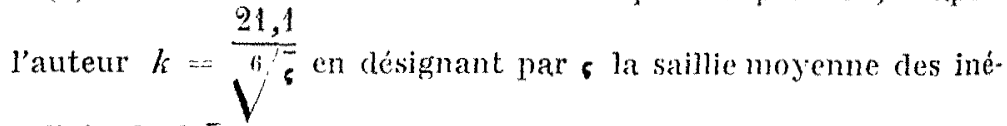
galités de la"surface des parois (ou la dimension, dans le sens normal à l'écoulement, des graviers constituant le lit d'un cours d'eau). 
Le coefficient c de Chézy anrail done pour expression :

$$
c \cdots=k \mathrm{R}^{\frac{1}{6}}
$$

La formule proposée, renarquablement simple -- strtout si on la compare à la grande fornule de Ganguillet et KuLter -. s'appliquerait à tous les cas rencontrés en hydraulique pratique, qu'il s'agisse de rivières naturelles, de canaux à écoulement libre, ou de conduites forcées. Ainsi que les autres formules, elle indiquerait que le coefficient de Chézy crôt avec le rayon moyen (Voir ligure 1). Toutefois, alors que, d'après les autres formules le cocfficient de Chézy tend vers une limile fixe, indépendante de la rugosilé, lorsque le rayon moyen augmente, de sorle que, pour un rayon assez grand, le débit deviendrait indépendant de la rugosilé, il n'en est. pas ainsi avec la tormule proposée; les débits, pour des sections géométriquement identiques et pour une même pente, resteraient dans les mêmes rapports que les coefficients de rugosité, et le coefficient de Chézy croît constamment avec le rayon.

Nous allons maintenant examiner sur quelles vérifications expérimentales est étayée la formule que nous examinons.

L'auteur a dépouillé les résultats de très nombreuses mesures de débit exécutées sur le Rhin, par la méthode du moulinet ou des flolteurs de surface. Nous citerons tout d'abord 17 mesures exéculées sur le Rhin à Bâle, dans lesquelles la pente superfi-

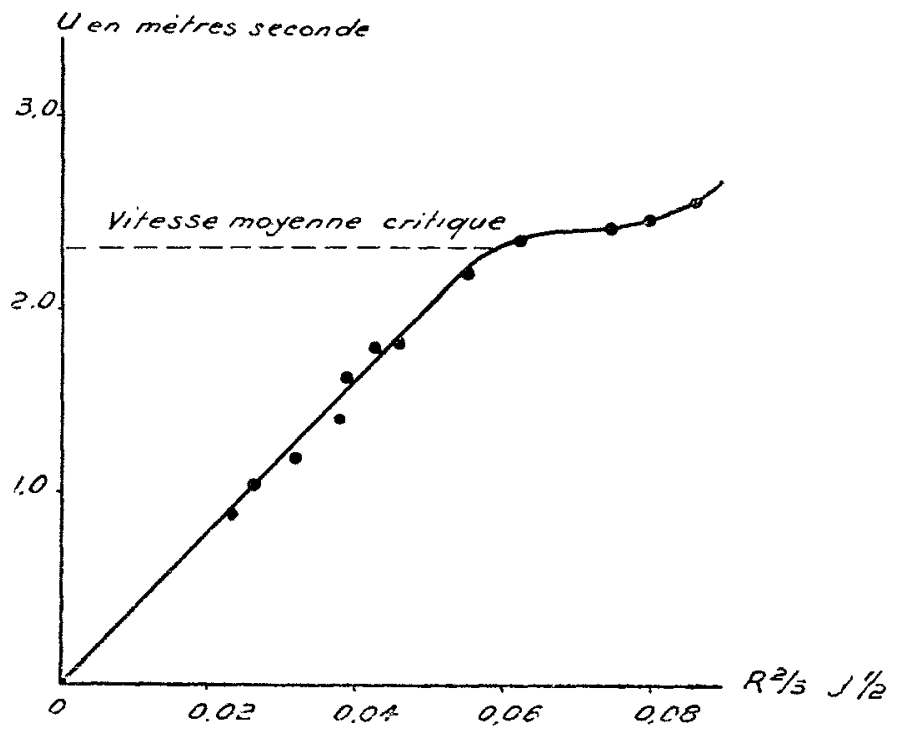

Fig. 2.- Le Rhin à Waldshut. - Point donné par 2 n 3 mesure de débit (en abscisse, la quantité $\mathrm{R} 2 / 3 ; 1 / 2$, en ordonnée la vitesse moyenne $u$.

cielle a varié de 0,00029 à 0,0011 et le rayon mouillé de $2 \mathrm{~m} .16$ à $7 \mathrm{~m} .14$ avec des variations corrélatives du débit comprises entre 325 et $5500 \mathrm{mc} / \mathrm{sec}$. On a obtenu, pour le coefficient $k$, des valeurs variant de 28,1 a 31,8 , et se groupant généralement entre 32 et 34,8 . Pour faire rentrer les mèmes résultats dans le cadre de la formule de Bazin, il ent fallu faire varier le coefficient 8 entre les valeurs 0,017 et 0,031 , ce coefficient croissant avec la vitesse moyenne dans le profil considéré.

On est d'ailleurs frappé, en faisant la part des multiples causes d'erreur inhérentes à toutes les opérations de jaugeage des rivières, de la parfaite concordance des résultats obtenus aux diverses stations du Rhin, et même de la grande analogie de l'ensemble de ces résultats avec ceux trouvés sur d'autres fleuves ou rivières.

Nous croyons ulile de grouper ci-après, de façon résumée, quelques-uns de ces résultats :

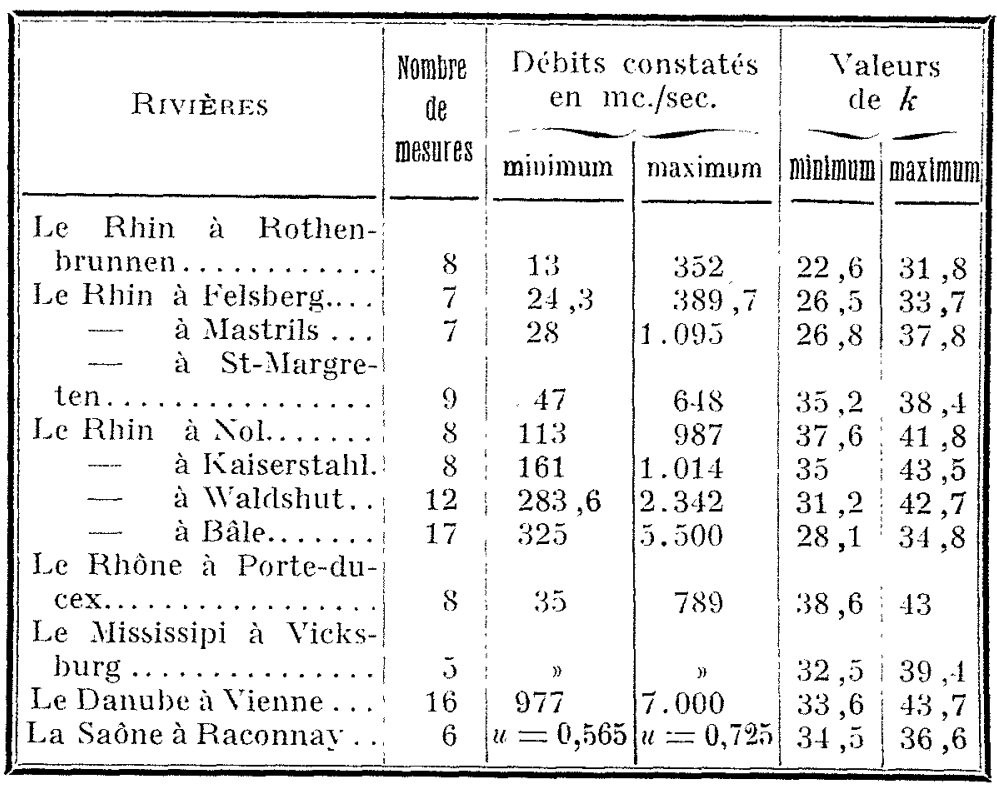

Il faut d'ailleurs remarquer que les valeurs de $k$ baissent pour la plupart des stations, assez régulièrement à partir d'une vitesse moyenne critique, qui, d'après l'auteur, est celle pour laquelle les matériaux constituant le fond commencent à ètre soulevés et cessent d'être posés à plat, ce qui a pour effet d'en augmenter la rugosité (1).

Si nous traçons la courbe des $u$ en fonction de la quantité $\mathrm{R}^{\frac{2}{3}} j^{\frac{1}{2}}$, on obtient une courbe analogue à celle ci-contre (fig. 2). La vitesse critique, pour le Rhin à Bâle et à Waldshut, est voisine de 2 m. 50 par sec.

Tous les résultats que nous venons de citer sont relatifs à des rivières à faible pente (variant de $\frac{2}{100.000} \grave{a} \frac{1}{1.000}$ ). D'autres résultats, en assez petit $n^{0}$ mbre, sont donnés sur la Lutschine, la Drance de Bagne, pour des pentes allant jusqu'à $\frac{56}{10.000}$; ils paraissent également satisfaisants. On peut regretter néanmoins que ces résultats ne soient pas plus nombreux, et ne concernent pas des torrents à plus forte pente. Nous savons, il est vrai, combien il est difficile de faire des mesures de pente et de section dans des lits souvent encombrés de rochers, créant des tourbillons et des remous, dont il est difficile de dégager l'influence.

Les expérimentations faites au moulinet sur les galeries d'amenée en souterrain, qui sont mentionnées dans le travail que nous analysons sont intéressanles, en raison de la pénurie

(1) La dimension 5 qui entre dans la formule $k=\frac{1}{6 / 5}$ se trouve majorée, donc le coefficient de Chézy diminue. 
des résultats publiés sur celte question: nous croyons utile? d'en domer ci-dessous un tableau résumé :

$$
c_{2}=\mathrm{R}^{\frac{1}{6}}==\left(\frac{\mathrm{D}}{t_{k}}\right)^{\frac{1}{3}} \times k
$$

\begin{tabular}{|c|c|c|c|c|c|c|c|c|c|}
\hline $\begin{array}{l}\text { DESIGNATIOA DE } \\
\text { IAA GALERIE }\end{array}$ & $\begin{array}{c}\text { NATURE DES } \\
\text { PAROIS }\end{array}$ & PENTE & $\begin{array}{c}\text { Nonluke } \\
\text { DE } \\
\text { Mesures }\end{array}$ & Serme & $\begin{array}{c}\text { Dimensiopls } \\
(b \times x) \\
\text { maxima }\end{array}$ & Vitesses & maxima & VAlame & 1) $k$ \\
\hline Sitterstollen..... & béton lissé & 0,000555 & 4 & ter à cheval & $2^{m} \times 1 m 85$ & 0,88 & 1,50 & 86,2 & 88,5 \\
\hline Muhleberg. . . . . . & béton arec enduit lisse & $0,000-157$ & 6 & & " & 2,02 & 13,6 & 62,1 & 76,3 \\
\hline Navizance..... & Maçonnerie avec enduit & $0,003 \cdot 1$ & 1 & Per à cheval & $2 \mathrm{~m} \times 2 \mathrm{~m}^{2} \mathrm{j}$ & 3,65 & ” & 87 & \\
\hline Simmezuleitung & béton avec enduit & $0,000+6$ & 1 & fer à cheval & $2 \mathrm{~m} 10 \times 2^{\mathrm{m} 7}$ & 1,31 & $n$ & 85,5 & \\
\hline Kallnach ....... & beton arec enduit & 0,00065 & 1 & fer à cheval & $5^{\mathrm{m}} \times 6^{\mathrm{m}}$ & 2,72 & $n$ & 87 & \\
\hline Martigny-Bourg & sans revêtement & 0,0025 & 1 & 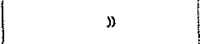 & " & 2,01 & $\eta$ & 52 & \\
\hline Ackersand..... & radier bétonné seul & 0,0025 & 1 & " & $"$ & 1,33 & $"$ & 49,2 & \\
\hline Gampel II. . & parois brutes & 0,0030 & 1 & $"$ & $"$ & 1,11 & $n$ & 10,8 & \\
\hline Biaschina. & galerie avec simple enduit & 0.0015 & 1 & $"$ & $n$ & 2,30 & $"$ & $6+, 1$ & \\
\hline
\end{tabular}

On constate, sur les deux premières galeries, une constance assez satisfaisante du coefficient $k$. Ici encore, on eût aimé que les essais fussent plus nombreux, particulièrement sur les galeries laissées sans revêtement.

Les résultats obtenus par Bazin sur les canaux artificiels, qui constituent encore l'expérimentation la plus précise faite sur les pertes de charge, n'ont pas été laissés de côté ; les expériences constituant les séries $24,25,39,7,8$ et 26 présentent une concordance très satisfaisante avec la formule de Strickler. Cela ressort du tableau ci-après :
Posons : $\quad / \overline{2} \mathrm{~A}=\mathrm{B}$, el $k=2^{\frac{7}{6}} \mathrm{~B}$

Nous aurons : $k=2^{\frac{\pi}{3}} \mathrm{~A}$, soit $k=3,17 \mathrm{~A}$

et :

$$
\begin{gathered}
c_{1}=\mathrm{B} \sqrt{1+3 \sqrt{\frac{\mathrm{D}}{\omega}}} \\
c_{9}=2\left(\frac{\mathrm{D}}{2}\right)^{\frac{1}{6}} \mathrm{~B}
\end{gathered}
$$

\begin{tabular}{|c|c|c|c|c|c|c|}
\hline SERIE D'Essats & $\begin{array}{c}\text { Nombre } \\
\text { De } \\
\text { Mesures }\end{array}$ & PExte & vitesses & moyen. & $\begin{array}{c}\text { Valeurs } \\
\text { de } k\end{array}$ & OBSERVATHONS \\
\hline 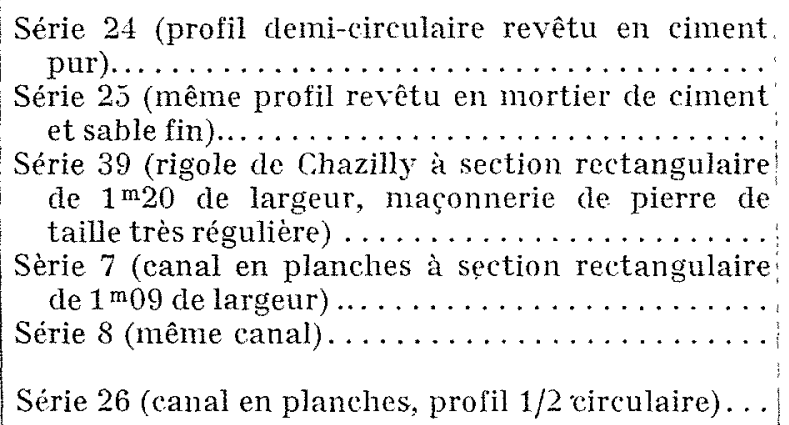 & $\begin{array}{c}4 \\
12 \\
12 \\
13\end{array}$ & $\begin{array}{c}0,00138 \\
0,0081 \\
0,00489 \\
0,00816\end{array}$ & $\begin{array}{l}0,875 \\
.1,71 \\
0,824 \\
0,938\end{array}$ & $\begin{array}{l}1,651 \\
2,68 \\
2,202 \\
2,707\end{array}$ & $\begin{array}{lll}97 & \text { à } & 106,5 \\
99 & \text { à } & 99,7 \\
78 & \text { à } & 81,9 \\
8-t & \text { à } & 86 \\
86 & \text { à } & 89,3 \\
81 & \text { à } & 86,5\end{array}$ & $\begin{array}{c}1 \text { résultat aberrant } k=65 \\
1 \text { résultat aberrant } \\
k=91, \bar{j}\end{array}$ \\
\hline
\end{tabular}

Sur les conduites circulaires, l'auteur a également fait l'analyse d'un grand nombre de résultats, notamment de ceux"oblenus par les expérimentateurs Fitzgerald, Iben, Marx-Wing-Hoskins, ainsi que dans le laboratoire de \%urich. Les résultats cités paraissent vérifier de façon très satisfaisante la formule proposée, qu'il s'agisse de conduites en fonte neufs ou incrustés, ou de tuyaux en bois raboté.

Il faut d'ailleurs remarquer que le coefficient de Chézy a sensiblement la même valeur si on emploie la formulede Maurice Lévy, ou si on utilise celle qui nous est proposée, à condition de prendre convenablement $\mathrm{A}$ et $k$. Ce coefficient a en effet pour expression avec la première formule :

et avec la seconde,

$$
c_{1}=V \overline{2} \times \sqrt{1+3 \sqrt{\frac{D}{2}}} \times A
$$


Nature des parois

Rochers très volumineux..............

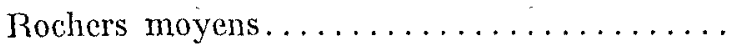

Rochers de la grosseur de la tête. . . . . . . . . Gravier grossier (environ $50 \times 100 \times 150 \mathrm{~mm}$.). Gravier moyen (environ $20 \times 40 \times 60 \mathrm{~mm}$.) .. Gravier fin (environ $10 \times 20 \times 40 \mathrm{~mm}$.)..... Gravier fin avec beaucoup de sable. Maçonneric

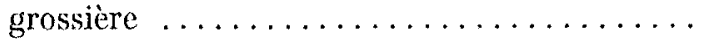

Bonne maçonnerie de moellons béton bien exé-

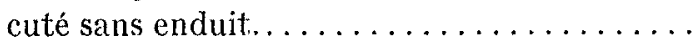

Pierre de taille. . . . . . . . . . . . . . . . .

Tuyaux de fonte neufs. Béton lissé. Planches de bois ............................

Tuyaux modérément incrustés . . . . . . . . . Bois bien raboté. Enduits lissés au ciment ....

Le rapide exposé qui précède ne saurait prétendre à remplacer la lecture de l'ouvrage original de M. l'Ingénieur Strickler, qui est assez concis pour être difficile à résumer. Il traite également d'autres sujets, dont l'intérêt n'est pas moindre pour l'hydraulicien, et que nous nous contenterons d'indiquer brièvement :

Détermination d'une formule de perle de charge entièrement générale, s'appliquant à tous les fluides, s'écoulant sous le régime turbulent ou sous le régime laminaire.

Distribution des vitesses dans une mème section pour différents profils.
Vérification du calcul de la couche de remous du barrage de Rheinfelden sur le Rhin.

Sur le point plus spécial que nous venons d'examiner, nos conclusions seront les suivantes :

$1^{0}$ D'ores et déjà les vérifications expérimentales données par M. Stricker sont assez complètes pour que la formule qu'il propose de remettre en honneur soit retenue. Elle a d'ailleurs le mérite de la simplicité, et est d'une application facile;

$2^{\circ}$ Parmi les vérifications données, un grand nombre concernent des fleuves et rivières à faible pente. Il serait toutefois très intéressant de disposer d'un plus grand nombre de vérifications relatives aux rivières ou torrents à forte pente, et aux galeries d'amenée, puisque la formule proposée prétend ètre universellement applicable. On peut, à notre avis, le désirer d'autant plus que les multiples réalisations hydroélectriques exécutées en Europe offrent un riche champ d'études à des investigations de cette nature. C'est aujourd'hui dans le domaine de la réalité industrielle que doivent, à notre avis, être principalement entreprises les recherches hydrauliques.

Au point de vue du développement de la science française, il serait fort désirable qu'un service officiel ou quasi-officiel s'occupât de centraliser les résultats déjà obtenus dans les installations françaises. Nous verrions même fort bien l'Etat imposer à ses concessionnaires, dans le cahier des charges de la concession, l'obligation de faire exécuter, par un bureau d'essais agréé par lui, l'essai complet de ses installations, comportant notamment la détermination précise des pertes de charge dans les divers organes d'amenée de l'eau. Nous croyons les industriels suffisamment avertis de l'intérêt de la question pour accepter cette obligation. D'ailleurs, il faut progresser pour vivre. 